\title{
Neurocysticercosis: still some unanswered questions
}

\author{
Neurocisticercoses: ainda questões não respondidas \\ Luís dos Ramos Machado
}

Departamento de Neurologia, Faculdade de Medicina,

Universidade de São Paulo, Sócio-

Diretor do Laboratório Spina-

França, Sao Paulo SP, Brazil.

Correspondence:

Luís dos Ramos Machado; Praça

Amadeu Amaral, 47 / conj. 33;

01327-010 São Paulo SP; Brasil.

E-mail: luisrmachado@globo.com

Conflict of interest:

There is no conflict of interest to

declare.

Received 04 April 2014

Accepted 11 April 2014

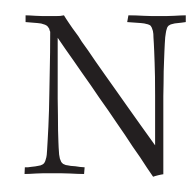

eurocysticercosis (NC), the cerebral infection with Taenia solium, is an important public health problem worldwide. Nevertheless, many important questions regarding NC remain unanswered, not only in relation to its etiopathogenesis and diagnosis but also in relation to its treatment.

In a magnificent text published in this issue of Arquivos de Neuro-Psiquiatria, Carpio, a world authority in studies on $\mathrm{NC}$, in collaboration with Romo ${ }^{1}$, masterfully bring their experience to bear on some important points that remain somewhat unclear in relation to this disease.

The natural history of cysticerci in the central nervous system (CNS) is not entirely understood $^{1}$. Their history is described more through information from computed tomography (CT) and magnetic resonance imaging (MRI) than from classical clinical or experimental models. A morphological classification based on the viability and location of the parasite in the CNS has been proposed: active, when the parasite is alive; transitional or degenerative, if it is in the colloidal or granular-nodular phase; or inactive, if there is evidence of its death ${ }^{2-4}$. Seizures are the main symptom in transitional parenchymal cysts ${ }^{1,5}$. However, these images are not pathognomonic of NC: (1) a single enhanced lesion on CT or hyperintense lesion on MRI is a common finding in patients with newly identified seizures in endemic countries; these patients, who are mainly children and young adults, have some benign and transitory clinical manifestations, predominantly focal seizures or focal seizures with secondary generalization; occasionally, they have Todd's paresis or focal neurological deficits $^{6}$; (2) these lesions may be seen in CNS tuberculosis and in other inflammatory pathological conditions ${ }^{1}$.

Seizures are the most common clinical manifestation of NC. Carpio and Romo emphasize that it is essential to distinguish between seizures and epilepsy in relation to neurocysticercosis ${ }^{1,5}$. Acute symptomatic seizures may result from transitional or degenerating cysts, but are ultimately a consequence of the acute inflammatory response (seizures, not epilepsy). In contrast, a patient with seizures who has viable cysts and/or inactive, non-inflamed calcified cysts may be categorized as having unprovoked or remote seizures (epilepsy) ${ }^{1,5}$. This difference is often difficult to assess. Although NC is one of the most frequent antecedents in patients with epilepsy, it may not necessarily be the main cause of epilepsy. It has consistently been reported in the literature that seizures are the most common symptom of NC, occurring in $70 \%$ to $90 \%$ of symptomatic patients. However, in a study on patients with newly-diagnosed epilepsy, perinatal brain damage (9\%), NC (8.3\%), other CNS infections (4.2\%), stroke (4.8\%) and head trauma (4.2\%) were the disorders most frequently reported as causes of epilepsy ${ }^{1}$.

The lack of validated diagnostic criteria for $\mathrm{NC}$ is another important problem ${ }^{1,4}$. This makes it difficult to estimate the prevalence of symptomatic NC. Additionally, because NC can be completely asymptomatic, this makes it almost impossible to estimate what proportion of the population will actually have symptomatic disease. Seropositivity through the enzyme-linked immunoelectrotransfer blot assay (EITB) or through the enzyme-linked immunosorbent assay (ELISA), a diagnostic criterion that has classically been accepted, does not necessarily imply the presence of active systemic infection with T. solium, nor does it 
imply CNS involvement ${ }^{1,4}$. In fact, the proportion of seropositivity in epileptic patients is similar to that reported in the general population in these same areas. Thus, the worldwide prevalence of $\mathrm{NC}$ remains unknown ${ }^{1}$.

Finally, Carpio and Romo point out very well, anthelminthic treatment for patients with seizures due to $\mathrm{NC}$ is not associated with improvement in remission from seizures ${ }^{1,7,8}$.

In summary, Carpio and Romo have compiled an excellent review on the current situation regarding NC. It provides in-depth reflection on a topic on which their knowledge is unmatched and to which they have dedicated the best part of their lives as researchers. They point out problems, open up perspectives and propose methodological changes. Furthermore, they raise awareness regarding erroneous designs and approaches in some of the classical studies that established not only diagnostic criteria but also protocols for treating NC. This review has all the credentials for leading a lucid, intelligent and modern reformulation of studies on NC.

\section{References}

1. Carpio A, Romo M. The relationship between neurocysticercosis and epilepsy: an endless debate. Arq Neuro-Psiquiatr 2014;72:383-390.

2. Carpio A, Fleury A, Hauser WA. Neurocysticercosis: Five new things. Neurol Clin Pract 2013;3:118-125.

3. Nash TE, Pretell EJ, Lescano AG, et al. Perilesional brain oedema and seizure activity in patients with calcified neurocysticercosis: a prospective cohort and nested case-control study. Lancet Neurol 2008;7:1099-1105.

4. Machado LR. The diagnosis of neurocysticercosis: a closed question? Arq Neuropsiquiatr 2010;68:1-2.

5. Carpio A, Hauser WA. Epilepsy in the developing world. Curr Neurol Neurosci Rep 2009;9:319-326.
6. Bansal BC, Dua A, Gupta R, Gupta MS. Appearing and disappearing CT scan abnormalities in epilepsy in India-an enigma. J Neurol Neurosurg Psychiatry 1989;52:1185-1187.

7. Romo ML, Carpio A, Kelvin EA. Routine drug and food interactions during antihelminthic treatment of neurocysticercosis: a reason for the variable efficacy of albendazole and praziquantel? J Clin Pharmacol 2014. [Epub ahead of print].

8. Carpio A, Kelvin EA, Bagiella E, et al Effects of albendazole treatment on neurocysticercosis: a randomised controlled trial. J Neurol Neurosurg Psychiatry 2008;79:1050-1055. 